\title{
Order Picking Performance : Strategies, Issues, and Measures
}

\author{
Byung Chun Park ${ }^{\dagger}$ \\ Department of Industrial and Management Engineering, Keimyung University, Daegu, 704-701, Korea \\ 오더피킹 성능 : 전략, 이슈, 측도 \\ 박 병 춘
}

\begin{abstract}
계명대학교 경영공학과
This paper is to review and organize performance strategies, issues, and measures for the efficient operation of order picking function. Order picking is the process of retrieving items from storage to meet a specific customer order, which is known to be the most labor-intensive and costly function among all the warehouse functions. This function is also important in that it has a critical impact on downstream customer service. For understanding the background of order picking and related performance issues, we will briefly introduce warehousing functions. Then we will introduce material handling within a warehouse and order picking strategies. Lastly, we will discuss about performance issues and measures in the domain of order picking operations. Productive and quality measures will be reviewed in more detail.
\end{abstract}

Keywords: Order Picking, Warehousing, Material Handling, Order Picking Strategies, Order Picking Performance Issues

\section{Introduction}

In modern business environments, warehousing functions are becoming important more than ever. Yet, such market's pressures as smaller shipments, faster delivery, and rapid proliferation of products make warehouse management difficult for meeting the demand for high service quality with low costs.

Material in a usual warehouse flows through the following functions :

- Receiving

- Put-away

- Storage

- Order picking

- Shipping.

Receiving is the function involved in receipts of materials coming into the warehouse. Put-away is the act of moving and placing materials in storage. The important decision in put-away is determining where and how much to store materials as there may be several storage modes or systems in most warehouses. By storage mode, we mean a region of storage area or a piece of storage equipment where the costs of picking from and restocking any location are all approximately equal (Bartholdi and Hackman, 2010). Example storage modes include pallet rack used for pallet storage, carton flow rack typically used for storing active cartons, and bin shelving typically used for storing slower, small loads.

Storage is to physically house material until requested. Storage systems can be classified into two types : unit load systems and small load systems. Unit load storage systems are used for housing large loads such as full pallets or large boxes. Block stacking or rack storage is a typical storage system used. Small load storage systems house small loads such as tote pans or small boxes, where the maximum capacity per storage location is typically less than $500 \mathrm{lbs}$ (Tomkins et al., 1996). Usual order picking is involved in picking from small load storage systems.

Order picking is the process of retrieving items from stor-

\footnotetext{
$\uparrow$ Corresponding author : Professor Byung Chun Park, Department of Industrial and Management Engineering, Keimyung University, 1000 Sindand-dong Dalseo-gu, Daegu, 704-701, Korea, Fax :+82-53-580-5287, E-mail : bcpark@kmu.ac.kr

Received August 20, 2011; Revision Received October 6, 2011; Accepted November 10, 2011.
} 
age to meet a specific customer order. Order picking function may include packaging of individual items or assortments. When an order consists of more than one item, it may be required to sort and/or accumulate items into individual orders, depending on the picking strategy employed. Shipping is involved in the activity of packing and accumulating orders by outbound carrier for loading.

Order picking is known to be the most labor-intensive and costly function among all the warehouse functions, typically accounting for $55 \%$ of warehouse operating costs (Tomkins et al., 1996). Order picking is also important in that it has a critical impact on downstream customer service. Customers expect quick and accurate processes of their orders, which is directly dependent on the efficiency of order picking operations.

Order picking systems can be classified largely into two types : picker-to-stock systems and stock-to-picker systems. In picker-to-stock systems, the picker travels by walking or riding to storage locations to retrieve items. In these systems, the travelling activity is the most expensive activity, typically accounting for $55 \%$ of order picker's time (Frazelle, 2002). An alternative solution to eliminate or reduce picker's travel is the stock-to-picker system. In typical stock-to-picker systems, the container or the storage location housing requested item is mechanically brought to the picker for retrieval. Thus, the picker's travel is replaced by the container's travel in stock-to-picker systems.

The purpose of this paper is to review and organize performance strategies, issues, and measures for the efficient operation of order picking function. Here, we are not intended to present a comprehensive review of the state-of-art order picking research. Instead, we are focusing on presenting a general introduction to order picking strategies, issues, and performance measures together with the state-of-art technology in order to give new insight into the problem. For that purpose, we will define warehousing terms explicitly in case of need. For a comprehensive review, please see Rouwenhorst et al. (2000) for warehouse design and control; de Koster et al. (2007) for design and control of warehouse order picking; Gua et al. (2007) for warehouse operations related to receiving, storage, order picking, and shipping; Baker and Canessa (2009) for a structured approach to warehouse design; and Gua et al. (2010) for warehouse design, performance evaluation, case studies, and computational support tools.

The organization of the study is as follows. For understanding the background of order picking and related performance issues, we briefly introduced warehouse functions. In section 2, we introduce material handling within a warehouse, where handling units and types of picks are explicitly defined. Then we review order picking strategies, where some warehousing terms are introduced more concretely. In section 3, we discuss about performance issues in order picking, including warehouse activity profiling, slotting and operating policies, and automation. In section 4 , we discuss about performance issues and measures in the domain of order picking operations. Productive and quality measures are discussed in more detail. Section 5 is devoted to concluding remarks.

\section{Order Picking}

\subsection{Material Handling within a Warehouse}

Material within a warehouse may be handled in pallet loads, in cases or cartons, in tote pans or bins, in inner packs, or even in individual pieces. Cases, cartons, tote pans, and bins are all containers that are used for holding items. A case is a container that is specially designed to hold or protect items. A carton refers to a rectangular cardboard box in use for storing items. A bin is a generic term referring to a container typically made of plastic, though metal bins are available. A tote pan in general refers to more standardized, reusable containers, typically used for in-process handling and stackable on top of one another. Both metal and plastic tote pans are available. So, we will not distinguish between bins and tote pans. Further, we will not distinguish between cases and cartons, if not in case of need.

$<$ Figure $1>$ shows the typical warehouse functions and flows. Depending on the types of retrieval units, types of picks can be classified into pallet pick, case pick, and broken-case pick. It is called a pallet pick when the picking quantity is a multiple of a full pallet load. We call it a case pick when the picking quantity is a multiple of a case quantity but less than a full pallet load. A broken-case pick, sometimes called a piece pick, is termed to represent an order pick where the picking quantity is less than a full case or in pieces.

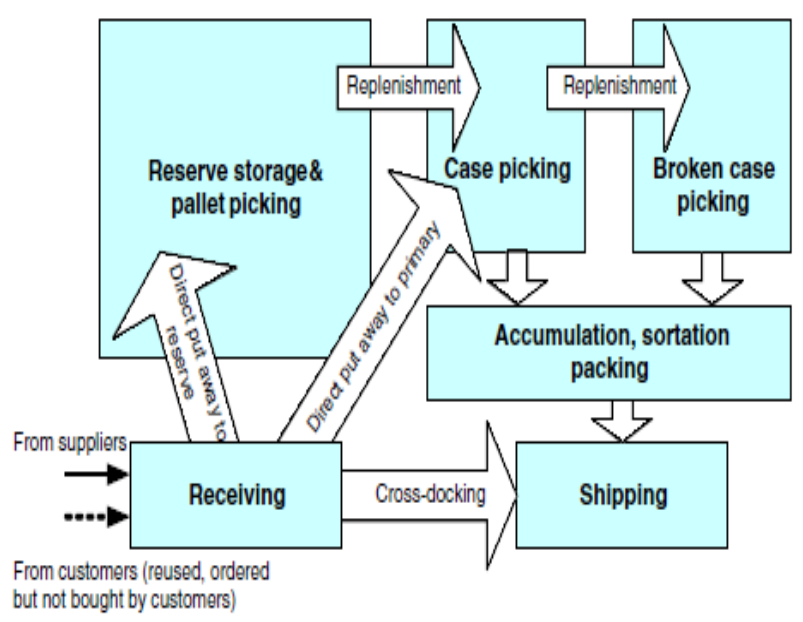

Figure 1. Typical warehouse functions and flows (Tomkins et al., 2003)

A pallet pick is typically done in unit load storage systems. Case pick can be done from pallet storage, bulk storage, or such case storage systems as carton flow racks. Usual bro- 
ken-case pick is done from small load storage systems, where items are commonly housed in cartons or bins. General characteristics of broken-case picks include a large number of item types, small quantities per pick, and short cycle times. Accordingly, broken-case picks are most costly and difficult to manage. Typically, order picking is involved in brokencase picks or case picks.

\subsection{Order Picking Strategies}

An order is simply a list of one or more order lines going to a specific customer or destination. An order line typically consists of a line item and its quantity requested. A line item is a separate item of supply on a transaction document. A stock keeping unit (sku) represents one unique inventory item in the smallest physical unit handled in a warehouse. Thus, line items and requested quantities should be expressed in terms of skus. Otherwise, communication error may arise.

Customer orders should be converted into pick lists for actual picking. A pick list is a list of one or more pick lines. A pick line typically consists of a line item, its quantity to be picked, and the storage location to be picked from. A pick list can be established from either only a single order or several orders. On the other hand, a single order can be broken up such that its line items appear in several pick lists. It is usual that the pick lines in a pick list are cleverly sequenced in order to reduce travel time, thereby improving the efficiency of picking operations.

It is called the single order picking if customer orders are completed one at a time. It is called the batch picking if orders are batched together so that all the line items requested in the batch of orders are picked from a location as it is visited by or presented to the picker. In other words, the order picker picks all orders within the batch using a consolidated pick list in a cycle. Usual batch sizes are from 4 to 12 orders, depending on the number of order lines per order.

In general, the single order picking is efficient for large orders with low demand, or emergency orders. By large order, we mean an order comprising of a large number of order lines, i.e., greater than ten order lines. Batch picking is efficient for small orders with high demand, let's say, high demand for orders of less than five-line items. The advantage of batch picking is that it can reduce travel time between picking locations by increasing pick density, the number of picks per unit of area (or per unit of travel length in one-dimensional systems). The disadvantage is that it requires additional work needed for order integrity, that is to say, sorting the picked items into individual customer orders.

The two typical approaches that are used for order integrity are sort-while-picking and sort-after-picking. In sort-whilepicking, the order picker sorts the line items into individual orders while picking in a cycle. Separate containers and/or special containers with several compartments may be used for order sortation/accumulation. In sort-after-picking, all the line items requested in the batch of orders are picked together during a picking cycle. The picked items are then sorted into individual orders in a downstream process. Batch picking is mostly used for broken-case picks. It can be used for case picks with sort-after-picking. It is rarely used in case picks with sort-while-picking, primarily because of its large physical size.

For the efficiency of picking operations, the picking area might be exhaustively divided into several picking zones. In picker-to-stock systems, a picking zone may consist of either a portion of a storage aisle, one aisle, or several aisles. In stock-to-picker systems, it could be a pod of retrieval equipments. It is called the zone picking if pickers are dedicated to a picking zone and responsible for picking items stored in that zone. Typically, zones are sized such that one or two pickers are assigned to a zone. It is called the free-form picking if pickers are free to pick from any location in the entire picking area. Zone pickings are frequently used along with batch pickings.

The primary objective of configuring zones in picker-tostock systems is to reduce travel times of pickers. A well-designed zone picking system can significantly reduce the picker's travel time. But, reduction in travel time can be offset by additional work required for order integrity. In stock-to-picker systems, the primary objective is to balance workload between the picker and retrieval equipments. In both systems, order integrity is a matter of concern.

The two popular methods used for order integrity in zone picking are progressive assembly and downstream sortation. In zone picking with progressive assembly, also called the pick-and-pass system, the contents of an order are passed from zone to zone in such containers as tote pans or cartons along a conveyor or on a cart until the order is completely assembled. Progressive assembly is most effective for systems with a large number of skus and for small to moderate orders with high demand.

In zone picking with downstream sortation, also called the wave picking, the order pickers work in their picking zones in parallel during a wave. In other words, picking operations are done in all zones at the same time. Typically, the order picker applies a bar code label to each unit picked. The picked item is placed into a large cart or onto a conveyor. It is then induced into a sortation system, where each customer order is completed. Wave picking requires a substantial investment on downstream sortation systems, and hence is suitable for systems with a high number of skus and heavy demand for moderate to large orders.

For zone picking to be successful, it is critical to balance the workload among zones, which is not an easy task. An alternative solution to progressive assembly with fixed zone sizes is to configure the picking area as a bucket brigade, a kind of progressive assembly with varying zone sizes. In a bucket brigade, zone sizes are automatically adjusted in order to keep balancing the workload among zones. For more detail on bucket brigades in a warehouse, see Bartholdi and Hackman (2010). 


\section{Performance Issues}

\subsection{Warehouse Activity Profiling}

In short, the mission of a warehouse is to meet the customer demand on higher service quality with lower costs. Thus, understanding the customer demand is the first step to be taken for the proper design and/or operation of order picking systems. This can be done by establishing warehouse activity profiling, which is the systematic analysis of warehouse activities. Example activity profiles include customer order profile, item activity profile, purchase order profile, and inventory profile.

Most information on customer demand can be identified by establishing the customer order profile. A typical customer order profile includes such information on order activity as number of orders per day, number of order lines per order, number of units (pallets, cases, or pieces) per order line, number of order units per day, etc. The customer order profile can serve as a valuable input to establishing other warehouse activity profiles such as item activity profile.

The statistical distribution of order activity is extremely useful not only for designing or reconfiguring a warehouse but also for improving warehouse activities. As an example, it can be used for encouraging customers to order in larger units, thereby reducing handling costs while improving service quality. It can also be used in receiving effectively for making orders and scheduling receipt. An example is in use for implementing cross-docking within a warehouse, where items move directly from receiving to shipping. With this approach, costly put-away, storage, and order picking can be eliminated.

The item activity profile shows activities of individual items in a warehouse, typically including popularity and volume in units handled for each item, handling characteristics, daily demand variation and seasonality, and demand correlation among line items. The popularity of an item can be measured in several ways, but the most important one to be used for warehouse management is the number of retrieval requests per unit time for the item. Handling characteristics of an item includes physical characteristics such as shape and weight, units to be handled, and environmental characteristics such as frozen, flammable, or hazardous material. The item activity profile can be used effectively for selecting proper storage or handling methods for each item.

\subsection{Slotting and Operating Policies}

By slotting, we mean the careful determination of storage place for each item. Specifically, it involves determining proper storage mode, space volume to be allocated, and specific storage location within a storage mode for each item. The whole quantity of the same item type can be stored in a specific storage mode, or distributed over several storage modes. The most information in use for slotting or reslotting the warehouse properly can be provided from the item activity profile. Slotting has a significant impact on put-away and order picking activities.

Performance issues closely related to slotting are layout configuration, pick-and-reserve problem, and restocking policy, among others. Layout configuration is involved in determining storage space requirements, dimension of handling units, number and dimension of aisles, layout of aisles, number and locations of input/output points, and takeaway/delivery systems. In most distribution warehouses, the forward pick area and the reserve storage area are separated. A forward pick area is a small sub-region of a warehouse, specially configured for efficient picking operations, where items are usually stored in small units. The reserve storage area is a large area for use in storage of items in large units, which are primarily used for restocking forward areas. The restocking policy is involved in determining what, when, where, how much to restock the forward area from the reserve area. All these have a great impact on the efficiency of picking operations.

Performance issues regarding operating policies include storage and retrieval policies. The storage policy is involved in determining the storage location within a storage mode for each item. Storage policies can be classified into random storage, dedicated storage, and class-based storage. Random storage is a generic term to represent the storage policy where the storage location is assigned to the item without considering its activity or turnover. When modelling random storage, each available location is assumed to be equally likely selected for storage, resulting in uniformly distributed activity over the locations. Selecting available locations in random, however, is cumbersome, and sometimes even costly. In practice, the closest open location rule is used. Under the closest open location rule, the closest, available location to the input/output point is selected for storage. Park and Lee (2007) showed that the assumption of uniform activity can be used fruitfully for modelling storage systems operated under the closest open location rule.

In dedicated storage, each item has a dedicated location or a fixed slot. Some items may be requested more frequently than others, thus having higher activity. We call the turnover-based storage if the storage location for each item is determined based on its turnover rate. In class based storage, the items are partitioned into several classes, typically based on their turnover rates. Then, a set of storage locations is assigned to each class. Random storage is used within a class.

The retrieval policy is involved in actual retrieval operations within a storage mode, and includes issues of retrieval time window, interleaving policy, and pick sequencing and path problem. A retrieval time window is the period of time during which requested orders are batched together to reduce travel time between picking locations. An example is the retrieval time window with zero time length, which results in the single order picking with first-come-first-serve basis. 
The interleaving policy is concerned with interleaving retrieval operations with storage operations. In some systems, retrieval requests are interleaved with storage requests such that both storage and retrieval operations are performed in a cycle. With this approach, the overall efficiency of an order picking system can be greatly improved. A miniload system is a typical example system where interleaving is commonly employed.

Given a set of pick lines, the pick sequencing problem is to determine the proper sequence of the picking locations to reduce travel time. In picker-to-stock systems, this problem is a kind of a travelling salesman problem, the problem of visiting a given set of locations as quickly as possible during a tour. The travelling salesman problem is extensively studied in the literature, yet no efficient, exact solution is reported (NP-hard). Consequently, heuristic procedures are commonly used. Clever pick sequencing can also reduce travel time dramatically in stock-to-picker systems (Bartholdi and Platzman, 1986; van den Berg, 1996; Litvak, 2006). The pick path problem is involved in determining the actual path to follow. Given a set of locations with a visiting sequence, there may be a number of different paths to visit the locations. Two most popular pick paths used in picker-to-stock systems are the serpentine pick path and the mainline path with side trips.

A serpentine path is the travel path that goes through in each storage aisle in an one-way direction. No turning back is allowed in the aisle. Clearly, travelling along the serpentine path, in the literal sense of the word, can result in a huge waste of time, unless the picker is required to visit every storage aisle during a tour. In mainline path with side trips, the picker travels along the main path that crosses the storage aisles, and detours into the aisle when necessary for picking. The advantage of this approach is that it can reduce travel time by storing more active items in locations close to the main path, thereby being able to decrease the number and length of the side trips. For more on pick-path optimization problems, see Ratliff and Rosenthal (1983) and Bartholdi and Hackman (2010).

Besides performance issues addressed here, there are other design and operational issues resolved for the fast, accurate, and efficient handling of customer orders. But, probably the most general principle of utmost importance would be that put-away, storage, and order picking should be considered simultaneously in both of design and operation. Clever putaway can greatly improve not only its efficiency but also order picking efficiency. As mentioned, slotting significantly impacts the efficiency of both put-away and order picking activities. In turn, innovative order picking strategy and system can eliminate or reduce well the need for put-away or storage. An example is picking from the reserve storage area. Frazelle (2002) reports the case of Ford's service parts distribution center, where the 54 carousels act as the reserve storage area while achieving forward picking rates by bringing reserve storage locations to picking stations.

\subsection{Automation}

Automation also greatly impacts on order picking performance. In general, there are two kinds of automation issues in a warehouse : one for physical handling systems and the other for information handling systems. Automation issues in physical handling systems include automation and integration of storage/retrieval operations and material flows together with information. They are involved in determining the type and technology of equipments and the degree of automation and integration.

In the domain of order picking operations, a usual approach to automation regarding physical handling systems is to facilitate automatic presentation of materials or information to the picker. High speed and accuracy of picking operations are essential elements for success in today's market environment, which demands fast delivery without error. In that context, this technology is becoming important more than ever, since it can significantly improve both picking productivity and accuracy.

Automatic presentation of materials to the picker is increasingly popular. A system may be configured such that the requested locations or containers are automatically brought to the picker. Example systems are miniload and carousel systems. The primary objective of employing this technology is to reduce or eliminate the picker's search and travel, the two most costly work elements of picking operation. This technology can also help the picker extract items without error.

Automatic presentation of information to the picker is also increasingly popular. The main direction is toward facilitating hand-free and paperless operation. Pick-to-light systems, voice-directed picking systems, and virtual displays are example technologies. In the context of picking operations, the primary advantage of employing these technologies is that these technologies can greatly facilitate efficient searching for and extracting items from the locations with less errors.

The pick-to-light system is a light-directed picking system, typically consisting of indicator lights and displays. It is used for presenting the picking location and quantity to be picked to the picker. It has a wide variety of applications. Example application systems include bin shelving, gravity flow racks, and carousels. In a typical application, a light display is placed at the front of each location. The light is illuminated if a pick is required from that location. In some systems, light displays can be integrated even into cartons or tote pans where picked items are placed, thereby being able to improve both picking efficiency and accuracy. The pick-to-light system is most popular in high volume picking.

In voice-directed picking systems, synthesized voice is used for giving instructions to the picker in real time. Voice technology is rapidly growing in recent years and is now a very viable solution for piece pick, case pick, or pallet pick operations. Lower cost than pick-to-light system, voice picking can deliver similar number of picks per hour. This system 
is also used effectively in high volume picking. In a virtual display-directed picking system, virtual display technology is used for directing the picking tour and/or giving instructions to the picker.

Information handling systems in a warehouse include the warehouse management system, communication system, and automatic identification systems. A warehouse management system is a kind of warehouse information system that tracks materials from receiving to shipping, manages warehouse activities and inventory, and processes or generates business data and information, and so on. Communication systems are interfaces that connect the warehouse management system to physical and information handling systems. Local area networks, radio frequency data communication and voice-based systems are example communication systems. Automatic identification systems are used for collecting relevant data automatically, which are then used for real time control of materials or operations. Example systems include bar coding, radio frequency, and vision systems.

Proper automation has several advantages. It can greatly reduce labor costs, increase inventory and picking accuracy, and shorten order cycle time. But, it usually requires a substantial investment. Furthermore, it is inflexible, i.e., it is more difficult to reconfigure the system to adapt to new business environments. Thus, to make it function properly and reliably, it should be well designed and integrated. To work effectively, it should also be accompanied with work simplification and standardization.

\section{Performance Measures}

System throughput capacity, system sojourn time, and picking accuracy are considered to be the three most important performance measures for order picking systems. Throughput capacity is the typical measure that is used for comparing performances of different order picking systems. System sojourn time and picking accuracy have a direct impact on downstream customer service.

\subsection{Financial and Productive Measures}

The performance of order picking systems can be measured from the financial point of view or the operational point of view. Financial measures are commonly used when needed to measure the system's performance in financial terms at the higher level. Usual financial measures are based on such data as costs, volume in units handled, and sales. Cost data can be collected for each of the warehouse activities and for each of the major resources consumed. Usual activity-based costing/management and benchmarking are very useful when measuring financial performances.

Since we are more interested in efficient operation of the order picking system, we focus on operational measures. A common measure used in measuring how well a system operates is productivity. Productivity is a kind of efficiency measure, and is defined to be the ratio of outputs divided by the inputs used. In the context of order picking operations, the outputs may be the number of completed orders, line items, transactions, etc. Usual inputs are such resources consumed as time, labor, space, energy, and money. An example productive measure is labor productivity, the ratio of the number of line items or orders completed to the number of labor hours spent.

A productive measure that is conveniently used in a warehouse is the storage density, the ratio of the amount of storage capacity available to the square footage used. The utilization of such key resources as machines or order pickers is another example of productivity measures. Since operation is mainly concerned about making the best use of resources available, productivity is a fundamental, operation-oriented performance measure.

In regard to performance of order picking operations, probably the most important productivity measure is the system throughput capacity or maximum throughput. By system throughput capacity, we will mean the 'maximum' long run average rate at which the system can process requests. Here the input is the period of time spent and the output is the number of requests completed. For the system operating under the stability condition, the system throughput is simply the demand rate. Thus, a matter of concern is to estimate the system throughput capacity. An approach for use in determining the throughput capacity is to model the system under the assumption that there exist an infinite number of requests to the system.

The efficient operation of an order picking system is critical under peak demand. Otherwise, it may become a bottleneck in an integrated warehouse system. Consequently, system designers are interested in the realization of maximum throughput, and consider the throughput capacity as the most important design criterion.

In order picking systems, throughput is typically measured in terms of pick rate. For broken-case pick, the pick rate is usually expressed in the number of pick lines picked per unit time (hour). For case pick, the pick rate can be expressed in either or both the number of cases and the number of pick lines picked per unit time. For pallet pick, the pick rate is usually expressed in the number of pallets picked per unit time. Throughput can also be measured in terms of number of orders completed per unit time. But, it must be careful to use this measure as a basis for comparison between two different systems, since it depends on the distribution of order sizes requested to the system.

\subsection{Quality Measures}

Resources consumed in the warehouse are important for warehouse management. But, it is not true for outside custo- 
mers. Usual customers are not concerned about resources consumed to meet their expectations. What they are concerned about are how well the system performs, how well it supports their requirements, etc. Quality measures are to evaluate how well a system functions.

Today's market pressure in a globally competitive environment makes quality measures increasingly important. From the progressive point of view, the quality of a system refers to the perception of the degree to which the system meets the customer's expectations. Thus, the quality of a system is determined by the customer, based upon the evaluation of his or her own entire experience. By system quality measures, we will mean the performance measures that are directly relevant to customer service. The two most important quality measures are system sojourn time and picking accuracy. Other quality measures include travel time and system queue size.

System sojourn time, also called system cycle time, is the elapsed time from when a request is released to the system until it is completed. Here, the request can be a pick line, a customer order, or a batch of customer orders. Again, it must be careful to use system sojourn time of an order as a basis for comparing performances of different order picking systems, because the performance depends on the distribution of order sizes. Furthermore, in some systems, the customer order may be completed in downstream sortation/accumulation system. Thus, it is very critical to clearly define the system boundary when comparing performances of different order picking systems.

System sojourn time is becoming more important under today's business environment that frequently demands the same day shipment. System sojourn time is also important from the operational point of view, since failure to correctly estimate the sojourn time of a request can cause starving or blocking at downstream operations, which will significantly affect customer service. In regard to warehouse performance, warehouse order cycle time measures the elapsed time from when an order is released to the warehouse until it is ready for shipping.

Picking accuracy is the percent of pick lines picked without error. Picking accuracy is a key measure of great concern to usual customers, since it directly impacts customer service. There are a variety of factors that impact picking accuracy in a warehouse, including human, equipment, material, method, and environment. A popular approach to improving picking accuracy is to employ such technologies as automatic identification and presentation.

Travel time is one of the issues extensively studied in the literature. For picker-to-stock systems, a matter of concern is the picker's travel time to the picking location. For stockto-picker systems, the matter is the travel time of the storage location or container to the picking station. Travel time and throughput are closely related. To increase throughput, travel time should be minimized. The usual approach to minimizing travel time is to sequence a set of the pick lines such that the sum of the individual travel times is minimized. But, a care must be exercised: for order picking systems, simply minimizing the travel time can not necessarily guarantee maximizing the throughput, as it depends upon the interaction with the picker. In fact, Park et al. (2006) showed that for miniload systems, minimizing the expected single or dual command travel time did not result in maximizing system throughput.

System queue size is the number of requests waiting for system service. System queue size and system sojourn time are also closely related. Large queue typically implies long sojourn time, and vice versa. Furthermore, large queues may indicate heavy congestion at interfaces or serious delays at downstream operations.

\subsection{Benchmarking}

Many warehouses use key performance indicators (KPI) to manage warehouse operations and to improve productivity. The KPIs are simply a set of several key performance indicators, typically representing operation costs, productivity, cycle time, or order accuracy. Based on the KPIs, warehouse performance gap analysis can be carried out, which is the graphical representation of a warehouse's performance in each performance dimension, compared to that of the industry best. The performance gap analysis can be used to identify weak or strong points in the performance of warehouse operations (Frazelle, 2002).

Benchmarking is the process of comparing one's products, business processes, or even strategies to those of the most successful firms in the same industry or from other industries. Dimensions typically measured are quality, time, and cost. Benchmarking gives a single efficiency score that can be used for comparing performance. There are several types of benchmarking. Metric benchmarking involves using more aggregative cost or operation information to identify strong and weak performing units. The two most common forms of quantitative analysis used in metric benchmarking are data envelope analysis and regression analysis.

Hackman et al. (2001) conducted a data envelope analysis to compare warehousing and distribution operations. The conclusions from their research are as follows. There was no definite difference in performance between union and nonunion warehouses, and between smaller warehouse and larger warehouse, respectively. Warehouses with low capital investment tend to outperform those with high capital investment.

\section{Concluding Remarks}

We reviewed and organized performance strategies, issues, and measures for the efficient operation of order picking 
function. We also defined some warehousing terms explicitly that were frequently used unclear in the literature. First, we briefly introduced warehousing functions, followed by material handling within a warehouse and order picking strategies. We then discussed about performance issues and measures, economical and operational, in the domain of order picking operations.

The topics not dealt with in this paper includes types of order picking systems, design issues, modelling issues, performance models, and some energy and environmental issues together with recent technology developments in order picking systems. An important design issue is to provide some guidelines for use in design and analysis of the system in early design phase. A modeling issue that deserves special attention is the interaction between picker's operations and storage system's operations.

\section{References}

Baker, P. and Canessa, M. (2009), Warehouse design : A structured approach, European Journal of Operational Research, 193(2), 425436.

Bartholdi, J. J. III, and Hackman, S. T. (2010), Warehouse and Distribution Science : Release 0.92, The Supply Chain and Logistics Institute, School of Industrial and Systems Engineering, Georgia Institute of Technology, Atlanta, GA 30332, USA.

Bartholdi J. J., III, and Platzman, L. K. (1986), Retrieval strategies for a carousel conveyor, IIE Transactions, 18(2), 166-173.

de Koster, R., Le-Duc, T., and Jan Roodbergen, K. (2007), Design and control of warehouse order picking : A literature review, European Journal of Operational Research, 182(2), 481-501.
Frazelle, E. H. (2002), World-Class Warehousing and Material Handling, McGraw-Hill, New York.

Gua, J., Goetschalckx, M., and McGinnis, L. F. (2007), Research on warehouse operation : A comprehensive review, European Journal of Operational Research, 177(1), 1-21.

Gua, J., Goetschalckx, M., and McGinnis, L. F. (2010), Research on warehouse design and performance evaluation : A comprehensive review, European Journal of Operational Research, 203(3), 539549.

Hackman, S. T., Frazelle, E. H., Griffin, P. M., Griffin, S. O., and Vlatsa, D. A. (2001), Benchmarking warehousing and distribution operations : An input-output approach, Journal of Productivity Analysis, 16(1), 79-100.

Litvak, N. (2006), Optimal picking of large orders in carousel systems. Operations Research Letters, 34(2), 219-227.

Park, B. C., Foley, R. D., and Frazelle, E. H. (2006), Performance of miniload systems with 2-class storage, European Journal of Operational Research, 170(1), 144-155.

Park, B. C. and Lee, M. K. (2007), Closest open location rule under stochastic demand, International J. of Production Research, 45(7), 1695-1705.

Ratliff, H. D. and Rosenthal, A. S. (1983), Order picking in a rectangular warehouse : A solvable case of the traveling salesman problem. Operations Research, 31(3), 507-521.

Rouwenhorst, B., Reuter, B., Stockrahm, V., van Houtum, G. J., Mantel, R. J., and Zijm, W. H. M. (2000), Warehouse design and control : Framework and literature review, European Journal of Operational Research, 122(3), 515-533.

Tomkins, J. J., White, J. A., Bozer, Y. A., Frazelle, E. H., Tanchoco, J. M. A., and Trevino, J. (1996), Facilities Planning, 2nd ed., John Wiley and Sons, Inc.

van den Berg, J. P. (1996), Multiple order pick sequencing in a carousel system : A solvable case of the rural postman problem, Journal of the Operational Research Society, 47(12), 1504-1515. 